\title{
Planning Singularity-free Force-feasible Paths on the Stewart Platform
}

Oriol Bohigas, Montserrat Manubens, and Lluís Ros

\begin{abstract}
This paper provides a method for computing force-feasible paths on the Stewart platform. Given two configurations of the platform, the method attempts to connect them through a path that, at any point, allows the platform to counteract any external wrench lying inside a predefined six-dimensional region. In particular, the Jacobian matrix of the manipulator will be full rank along such path, so that the path will not traverse the forward singularity locus at any point. The path is computed by first characterizing the force-feasible $\mathrm{C}$-space of the manipulator as the solution set of a system of equations, and then using a higher-dimensional continuation technique to explore this set systematically from one configuration, until the second configuration is found. Examples are included that demonstrate the performance of the method on illustrative situations.
\end{abstract}

Key words: Singularity-free path planning, higher-dimensional continuation, singularity avoidance, Stewart platform.

\section{Introduction}

Singular configurations of the Stewart platform are well-known and widely studied in the Literature $[9,11]$. They yield uncontrollable motions or unresolvable endeffector forces and, thus, they are to be avoided in the vast majority of applications. On this regard, several works consider the issue of local singularity avoidance [1], but only a few tackle the more general problem of computing singularity-free paths between two distant configurations. Solutions to this problem include an algorithm based on deforming a parametrized path between the query configurations [5], a variational approach that reduces the problem to a boundary value problem [14], and a numerical technique based on treating the singularity locus as a collection

O. Bohigas, M. Manubens, and L. Ros,

Institut de Robòtica i Informàtica Industrial, e-mail: \{obohigas,mmanubens,lros\}@iri.upc.edu. 
of obstacles [6]. All of these algorithms work well in favorable situations, but [5] and [14] mention limitations relative to proving path existence in certain cases, and [6] is computationally intensive, as it requires constructing polytope approximations of the entire singularity set before searching for the path. An important drawback of $[5,14,6]$, moreover, is that the clearance of the path relative to the singularity locus is measured in terms of the determinant or the condition number of the Jacobian matrix, which, as noted in [15], lack physical significance.

In contrast to such works, this paper provides a method for planning paths that maintain a physically meaningful clearance with respect to the singularity locus. This clearance is defined following the spirit of $[3,8]$, as the one that results from only allowing force-feasible configurations; i.e., those on which the leg forces remain within the allowed limits, for any platform wrench lying inside a prescribed six-dimensional region (Section 2). The planning method relies on defining a system of equations whose solution manifold corresponds to the force-feasible subset of the C-space, so that maneuvering through such manifold guarantees singularity avoidance at all times (Section 3). Then, an extension of the higher-dimensional continuation strategy given in [7] is defined to explore this manifold systematically, until a path joining the start and goal configurations is found, or path non-existence is determined at a given resolution (Section 4). The approach has been implemented and validated on several experiments (Section 5).

\section{Problem statement}

The Stewart platform consists of a moving plate, or platform, connected to a fixed base by means of six legs, where each leg is a universal-prismatic-spherical chain. The most general version of such manipulator follows the so-called 6-6 design, where the leg anchor points are all different (Fig. 1), though not necessarily coplanar [10]. The six prismatic joints are actuated, allowing to control the six degrees of freedom of the platform, and the remaining joints are passive.

Let $O X Y Z$ and $P X^{\prime} Y^{\prime} Z^{\prime}$ be fixed and moving reference frames, respectively attached to the base and the platform (Fig. 1). Any configuration of the platform can be uniquely

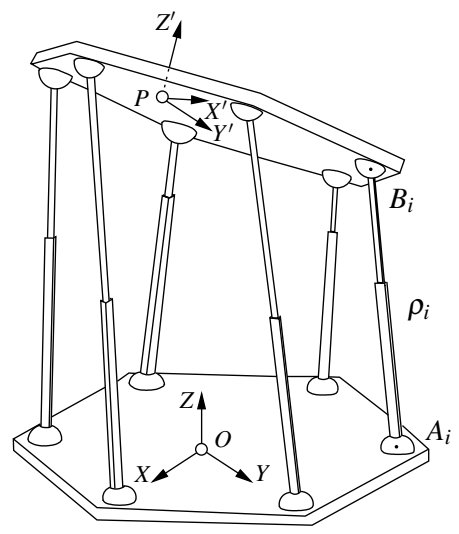
represented by a pair $\boldsymbol{q}=(\boldsymbol{p}, \boldsymbol{R}) \in S E(3)$, where $\boldsymbol{p}=[x, y, z]^{\top}$ is the position vector of point $P$ in the fixed frame, and $\boldsymbol{R}$ is a $3 \times 3$ rotation matrix providing the orientation of $P X^{\prime} Y^{\prime} Z^{\prime}$ relative to $O X Y Z$. Not all values for $\boldsymbol{R}$ and $\boldsymbol{p}$ are permitted though, because the leg lengths $\rho_{i}$ need to be within the range $\left[\rho_{i}, \overline{\rho_{i}}\right]$ of allowable values on each leg. Thus, if $\boldsymbol{a}_{i}$ and $\boldsymbol{b}_{i}$ denote the position vectors of the anchor points 
$A_{i}$ and $B_{i}$ of the $i$ th leg (Fig. 1), expressed in $O X Y Z$ and $P X^{\prime} Y^{\prime} Z^{\prime}$ respectively, the configuration will only be valid if for $i=1, \ldots, 6$ it satisfies

$$
\begin{aligned}
\rho_{i}^{2} & =\left|\boldsymbol{p}+\boldsymbol{R} \boldsymbol{b}_{i}-\boldsymbol{a}_{i}\right|^{2}, \\
\rho_{i} & \in\left[\underline{\rho_{i}}, \overline{\rho_{i}}\right] .
\end{aligned}
$$

Often, moreover, each configuration $\boldsymbol{q}$ must be force feasible, in the sense that the platform must be able to equilibrate any external wrench $\hat{\boldsymbol{w}}$ acting on it, subject to lie inside a given six-dimensional region $\mathscr{W} \subset \mathbb{R}^{6}$. The significance of $\mathscr{W}$ depends on the particular context of application. For example, $\mathscr{W}$ may be determined by the set of allowable inertia forces acting on the platform, or by the set of wrenches that should be suppliable to the environment. Specifically, the force-feasibility requirement on a given $\boldsymbol{q}$ implies that for any wrench $\hat{\boldsymbol{w}} \in \mathscr{W}$ there must be a vector $\boldsymbol{f}=\left[f_{1}, \ldots, f_{6}\right]^{\top}$ of leg forces satisfying

$$
\boldsymbol{J}(\boldsymbol{q}) \cdot \boldsymbol{f}=\hat{\boldsymbol{w}}
$$

with

$$
\boldsymbol{f} \in \mathscr{D}=\left[\underline{f_{1}}, \overline{f_{1}}\right] \times \ldots \times\left[\underline{f_{6}}, \overline{f_{6}}\right],
$$

where $\boldsymbol{J}(\boldsymbol{q})$ is the $6 \times 6$ screw Jacobian of the manipulator at configuration $\boldsymbol{q}$, and $\left[f_{i}, \overline{f_{i}}\right]$ is the interval of force magnitudes that can be resisted by the $i$ th leg. In this paper, $\mathscr{W}$ will be a six-dimensional non-degenerate ellipsoid defined by

$$
\left(\hat{\boldsymbol{w}}-\hat{\boldsymbol{w}}_{0}\right)^{\top} \boldsymbol{E}\left(\hat{\boldsymbol{w}}-\hat{\boldsymbol{w}}_{0}\right) \leq 1,
$$

where $\hat{\boldsymbol{w}}_{0}$ is a fixed wrench and $\boldsymbol{E}$ is a constant $6 \times 6$ positive-definite symmetric matrix. Also, $\hat{\boldsymbol{w}}, \hat{\boldsymbol{w}}_{0}$, and $\boldsymbol{J}(\boldsymbol{q})$ will be assumed to be given in a frame $P X Y Z$ centered in $P$ and parallel to $O X Y Z$, but any other frame could be assumed if desired.

Now, let $\mathscr{C}$ be the set of all $\boldsymbol{q} \in S E$ (3) satisfying Eqs. (1)-(4) for all $\hat{\boldsymbol{w}} \in \mathscr{W}$, which we will call the force-feasible $C$-space of the manipulator. Given two configurations in $\mathscr{C}, \boldsymbol{q}_{1}$ and $\boldsymbol{q}_{2}$, the goal of this paper is to provide an algorithm for computing a path on $\mathscr{C}$ connecting them, if one exists, or to determine path non-existence otherwise. To find such a path, we next define a system of equations that characterize $\mathscr{C}$.

\section{Equations of the force-feasible $\mathrm{C}$-space}

Let $\boldsymbol{f}_{0}$ be any vector of resultant leg forces corresponding to $\hat{\boldsymbol{w}}_{0}$, i.e.,

$$
\boldsymbol{J}(\boldsymbol{q}) \cdot \boldsymbol{f}_{0}=\hat{\boldsymbol{w}}_{0}
$$

By substitution of $\hat{\boldsymbol{w}}-\hat{\boldsymbol{w}}_{0}=\boldsymbol{J}(\boldsymbol{q})\left(\boldsymbol{f}-\boldsymbol{f}_{0}\right)$ into Eq. (5) we realise that, for a given $\boldsymbol{q}$, the set $\mathscr{F}$ of leg forces $\boldsymbol{f}$ satisfying Eq. (3) for some $\hat{\boldsymbol{w}} \in \mathscr{W}$ is given by $\left(\boldsymbol{f}-\boldsymbol{f}_{0}\right)^{\top} \boldsymbol{B}\left(\boldsymbol{f}-\boldsymbol{f}_{0}\right) \leq 1$, where $\boldsymbol{B}=\boldsymbol{J}(\boldsymbol{q})^{\top} \boldsymbol{E} \boldsymbol{J}(\boldsymbol{q})$. Hence, $\mathscr{F}$ is an ellipsoid, be- 
cause $\boldsymbol{E}$ is symmetric and positive-definite, but this ellipsoid will be bounded in all directions, or only in some, depending on whether $\operatorname{det}(\boldsymbol{J}(\boldsymbol{q})) \neq 0$ or not.

Now note that, for $\boldsymbol{q}$ to be force-feasible, it must be $\mathscr{F} \subset \mathscr{D}$, which can be checked as follows. Let $\boldsymbol{v}_{i} \in \mathbb{R}^{6}$ be a vector satisfying

$$
\begin{array}{r}
\boldsymbol{B}^{i} \boldsymbol{v}_{i}=\mathbf{0} \\
\boldsymbol{v}_{i}^{\top} \boldsymbol{B} \boldsymbol{v}_{i}=1 \\
v_{i, i} \geq 0
\end{array}
$$

where $v_{i, i}$ denotes the $i$ th component of $\boldsymbol{v}_{i}$, and $\boldsymbol{B}^{i}$ stands for the matrix $\boldsymbol{B}$ with its $i$ th row removed. If $\operatorname{det}(\boldsymbol{J}(\boldsymbol{q})) \neq 0$, then $\boldsymbol{B}$ and $\boldsymbol{B}^{i}$ are full row rank, and there is exactly one vector $\boldsymbol{v}_{i}$ satisfying (7)-(9). Using Lagrange multipliers, one can see that in such a case $f_{0}-\boldsymbol{v}_{i}$ and $f_{0}+\boldsymbol{v}_{i}$ are the vectors in $\mathscr{F}$ attaining the smallest and largest value along the $i$ th coordinate. Hence, when $\operatorname{det}(\boldsymbol{J}(\boldsymbol{q})) \neq 0, \mathscr{F} \subset \mathscr{D}$ iff

$$
f_{0, i}-v_{i, i} \geq \underline{f_{i}} \text { and } f_{0, i}+v_{i, i} \leq \overline{f_{i}}, \quad \text { for } i=1, \ldots, 6 .
$$

When $\operatorname{det}(\boldsymbol{J}(\boldsymbol{q}))=0$, it will always be $\mathscr{F} \not \subset \mathscr{D}$, because $\mathscr{F}$ will be unbounded along some of its principal directions.

Observe that the constraints in (2), (9), and (10) are equivalent to imposing

$$
\begin{array}{r}
\left(\rho_{i}-m_{i}\right)^{2}+r_{i}^{2}=h_{i}^{2}, \\
v_{i, i}=s_{i}^{2}, \\
f_{0, i}-v_{i, i}=t_{i}^{2}+\underline{f_{i}}, \quad f_{0, i}+v_{i, i}=-u_{i}^{2}+\overline{f_{i}},
\end{array}
$$

respectively, where $m_{i}$ and $h_{i}$ are the midpoint and half-range of $\left[\rho_{i}, \overline{\rho_{i}}\right]$, and $r_{i}, s_{i}$, $t_{i}$, and $u_{i}$ are newly-defined auxiliary variables. As a result, $\mathscr{C}$ can be characterized as the set of points $\boldsymbol{q}$ that satisfy the system formed by Eqs. (1), (6), (7), (8), and (11)-(13) for some value of the remaining variables. For ease of explanation, this system will be written as $\boldsymbol{F}(\boldsymbol{x})=\mathbf{0}$, where $\boldsymbol{x} \in \mathbb{R}^{n_{x}}$ encompasses all variables in the system, including those in $\boldsymbol{q}$.

Let $\mathscr{M}$ be the set of points $\boldsymbol{x}$ that satisfy $\boldsymbol{F}(\boldsymbol{x})=\mathbf{0}$, which is a manifold of dimension $d=6$ when no further constraints are imposed on $\boldsymbol{q}=(\boldsymbol{p}, \boldsymbol{R})$. Note that such points are in correspondence with the points $\boldsymbol{q} \in \mathscr{C}$ because any value of $\boldsymbol{q} \in \mathscr{C}$ determines the values for the remaining variables in $\boldsymbol{x} \in \mathscr{M}$. Thus, since $\boldsymbol{q}_{1}$ and $\boldsymbol{q}_{2}$ have corresponding points $\boldsymbol{x}_{1}$ and $\boldsymbol{x}_{2}$ on $\mathscr{M}$, and all paths on $\mathscr{C}$ are represented in $\mathscr{M}$, and viceversa, the original problem of computing a force-feasible path in $\mathscr{C}$ from $\boldsymbol{q}_{1}$ to $\boldsymbol{q}_{2}$ can be reduced to that of connecting $\boldsymbol{x}_{1}$ and $\boldsymbol{x}_{2}$ through a path on $\mathscr{M}$. It is not difficult to see, moreover, that for any $\boldsymbol{x} \in \mathscr{M}$ it will be $\operatorname{det}(\boldsymbol{J}(\boldsymbol{q})) \neq 0$, so that any path computed on $\mathscr{M}$ will be free of singular configurations. Certainly, if it were $\operatorname{det}(\boldsymbol{J}(\boldsymbol{q}))=0$ for some $\boldsymbol{x} \in \mathscr{M}$, then $\boldsymbol{B}$ would be rank deficient, implying that $\operatorname{Ker}\left(\boldsymbol{B}^{i}\right)=\operatorname{Ker}(\boldsymbol{B})$ for some $i$. Therefore, any $\boldsymbol{v}_{i}$ satisfying Eq. (7) would violate Eq. (8), which is in contradiction with the fact that $\boldsymbol{x} \in \mathscr{M}$. 

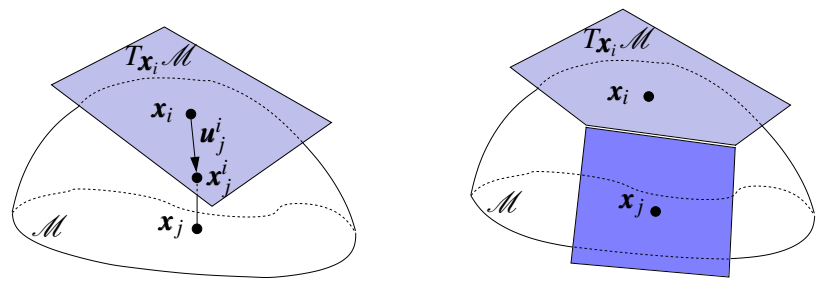

Fig. 2 The higher-dimensional continuation method applied to a 2-dimensional manifold in $\mathbb{R}^{3}$.

\section{Exploring the force-feasible C-space for a path}

To determine a path on $\mathscr{M}$ connecting $\boldsymbol{x}_{1}$ and $\boldsymbol{x}_{2}$ we can gradually construct an atlas of $\mathscr{M}$, i.e., a collection of charts where each chart $C_{i}$ defines a local map from a domain $\mathscr{P}_{i} \subset \mathbb{R}^{d}$ to an open set of $\mathscr{M}$ around a point $\boldsymbol{x}_{i} \in \mathscr{M}$, initially $\boldsymbol{x}_{1}$. The atlas will be computed using the higher-dimensional continuation approach proposed in [7], which defines the local map for chart $C_{i}$ using $\boldsymbol{\Psi}_{i}$, an orthonormal basis of $T_{\boldsymbol{x}_{i}} \mathscr{M}$, the $d$-dimensional tangent space of $\mathscr{M}$ at $\boldsymbol{x}_{i}$. The map is defined by first selecting a vector $\boldsymbol{u}_{j}^{i} \in \mathbb{R}^{d}$ of parameters (Fig. 2, left), which is used to generate a point $\boldsymbol{x}_{j}^{i} \in \mathbb{R}^{n_{x}}$ in the neighborhood of $\boldsymbol{x}_{i}$, using

$$
\boldsymbol{x}_{j}^{i}=\boldsymbol{x}_{i}+\boldsymbol{\Psi}_{i} \boldsymbol{u}_{j}^{i}
$$

Then, a point $\boldsymbol{x}_{j} \in \mathscr{M}$ corresponding to the projection of $\boldsymbol{x}_{j}^{i}$ on $\mathscr{M}$ is computed, by solving the system formed by $\boldsymbol{F}\left(\boldsymbol{x}_{j}\right)=\mathbf{0}$ and $\boldsymbol{\Psi}_{i}^{\top}\left(\boldsymbol{x}_{j}-\boldsymbol{x}_{j}^{i}\right)=\mathbf{0}$ using a Newton method initialized at $x_{j}^{i}$. Each point $\boldsymbol{x}_{j}$ is the potential center of a new chart (Fig. 2, right), and Henderson introduced a method to determine how to select the chart centers to ensure a good coverage of the manifold [7]. In his approach, the domain $\mathscr{P}_{i}$ of chart $C_{i}$ is initialized as a $d$-dimensional hypercube enclosing a ball $\mathscr{B}_{i}$ of radius $r$, both defined in $T_{\boldsymbol{x}_{i}} \mathscr{M}$, as illustrated in Fig. 3 , top. A vertex of $\mathscr{P}_{i}$ exterior to $\mathscr{B}_{i}$, with position vector $\boldsymbol{s}$, is used to generate a point $\boldsymbol{x}_{j}^{i}$, using (14) with $\boldsymbol{u}_{j}^{i}=\alpha \cdot \boldsymbol{s} /\|\boldsymbol{s}\|$, where $\alpha$ is initialized to $r$. If the projection of $\boldsymbol{x}_{j}^{i}$ to $\mathscr{M}$ does not con-
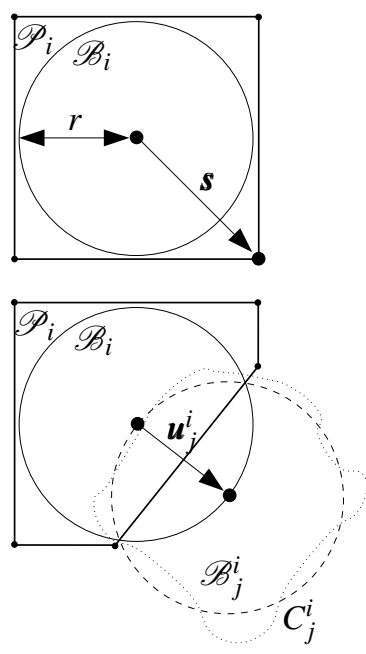

Fig. 3 Chart construction. verge, or if the new chart $C_{j}$ at $\boldsymbol{x}_{j}$ is too far or too different from $C_{i}$, the new chart is discarded and a new attempt of chart generation is performed with a smaller $\alpha$, allowing to adapt the size of the area covered by each chart to the local curvature of the manifold. When $C_{j}$ is valid, it is used to crop $\mathscr{P}_{i}$ from the intersection between $\mathscr{B}_{i}$ and $C_{j}^{i}$, the projection on $T_{\boldsymbol{x}_{i}} \mathscr{M}$ of the part of the manifold covered by $C_{j}$. This projection is approximated by a ball $\mathscr{B}_{j}^{i}$ of radius $r$ in $T_{\boldsymbol{x}_{i}} \mathscr{M}$, centered at the point 
given by $\boldsymbol{u}_{j}^{i}$, as shown in Fig. 3 , bottom. The intersection of $\mathscr{B}_{i}$ and $\mathscr{B}_{j}^{i}$ defines a new face for $\mathscr{P}_{i}$ that eliminates some of its vertices (in particular the one given by $\boldsymbol{s}$ ) and generates new ones. Symmetrically, the polytope $\mathscr{P}_{j}$ associated with $C_{j}$ is cropped using $C_{i}$. When $C_{i}$ is surrounded by other charts, $\mathscr{P}_{i}$ becomes a convex polytope included in $\mathscr{B}_{i}$, and $C_{i}$ is considered to be closed, meaning that no further expansion of the atlas needs to be attempted from that chart. When all charts are closed, the connected component of $\mathscr{M}$ containing the initial point $\boldsymbol{x}_{1}$ gets fully covered. If a path exists from $x_{1}$ to $x_{2}, x_{2}$ must be included in one of the charts of the atlas and, thus, a solution path can be determined by searching on the graph implicitly defined by the chart centers and their neighborhood relations. In practice, however, the expansion of the charts is performed according to an $\mathrm{A}^{*}$ search strategy using an admissible heuristic [13], so that the path is returned as soon as it is found without computing the whole atlas, and it is guaranteed to be the shortest possible on $\mathscr{M}$. If $x_{2}$ is not included in any of the charts in the end, path non-existence is established at the considered value for $r$.

\section{Experiments}

The method has been implemented in $\mathrm{C}$, and run on a iMac equipped with a 2.93 $\mathrm{GHz}$ Intel Core $\mathrm{i} 7$ processor. To verify its performance on a realistic situation, the geometric parameters of the INRIA left hand have been used [10]. All legs of this manipulator admit forces in the range $\left[f_{i}, \overline{f_{i}}\right]=[-300,300]$, and for the experiments we have set $\hat{\boldsymbol{w}}_{0}=[0,0,150,0,0,0]^{\top}$ and $\boldsymbol{E}=\boldsymbol{I}_{6 \times 6}$, assuming SI units throughout. In this case, the force-feasible $\mathrm{C}$-space of this manipulator is close to the workspace defined by its allowable leg lengths [10], and the algorithm solves typical planning queries in a few seconds, even when permitting the variation of all pose parameters.

To graphically illustrate the performance of the method, however, it is better to adopt the geometric parameters of the more academic manipulator in [9], where large variations of the leg lengths are allowed, leading to a very large workspace with interesting singularity surfaces. Two experiments are shown for this manipulator, assuming $\left[f_{i}, \bar{f}_{i}\right]=[-300,300]$ and $\boldsymbol{E}=\boldsymbol{I}_{6 \times 6}$ as before, but using $\hat{\boldsymbol{w}}_{0}=[0,0,1,0,0,0]^{\top}$. In a first experiment we compute a force-feasible path for the platform moving at a constant orientation, defined by the Euler angles $\phi=-2^{\circ}$, $\theta=30^{\circ}$ and $\psi=-87^{\circ}$ under the convention in [9], and at a constant value of $z$. Using the start and goal configurations defined by the positions $\boldsymbol{p}_{1}=[0.4,0,0.1]^{\top}$ and $\boldsymbol{p}_{2}=[-0.3,0,0.1]^{\top}$ for $P$, the resulting path in the XY plane is computed in 578 seconds. Fig. 4, left shows this path, together with the singularity curve to be avoided, the atlas corresponding to the whole force-feasible connected component accessible from $\boldsymbol{p}_{1}$ (shown as a mesh), and the region explored by the $\mathrm{A}^{*}$ algorithm (shaded in grey). It can be seen that the interpolated path between $\boldsymbol{p}_{1}$ and $\boldsymbol{p}_{2}$ would go through singularities, but the computed path correctly avoids them while keeping the leg forces within the specified ranges (Fig. 4, bottom). In a second experiment, we solve the same planning query but keeping constant the orientation of the plat- 


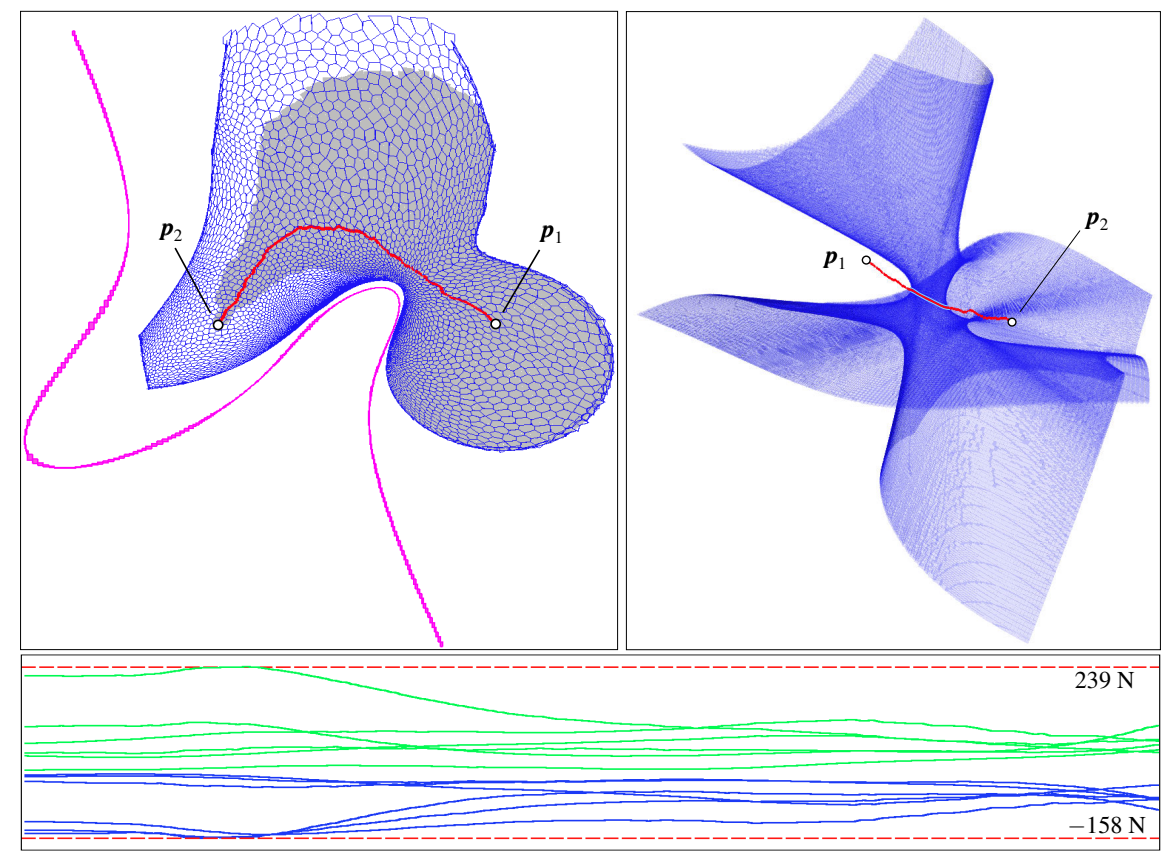

Fig. 4 Top: Two paths computed for the manipulator in [9]. Bottom: Plot of the maximum and minimum forces supported by each leg along the left path.

form only, obtaining the path in XYZ shown in Fig. 4, right in 90 minutes. The singularity surface, computed using [2] and shown in the figure, is correctly avoided by the computed path. It must be noted that these are hard planning queries, since the workspace in [9] is enormous when compared to typical workspaces arising in usual platforms. Moreover, once a partial atlas is computed, all planning queries between configurations covered by such atlas can be solved in a few milliseconds.

\section{Conclusions and future work}

This paper has presented a path planning method for computing non-singular paths on the Stewart platform, based on imposing the resolvability of a six-dimensional set of wrenches at any point on the path. The method has been tested succesfully on manipulators of various geometries, and computes paths in reasonable times in realistic situations. The presented approach could be generalized by requiring the path to fulfill additional constraints, like guaranteeing a certain positioning accuracy of the platform, or the avoidance of platform collisions. While the former constraints can in principle be incorporated using dual developments to those herein presented, the latter require investigating the possibility of randomizing the planner, in the spirit of [4] or [12], for example. 


\section{Acknowledgements}

This work has been partially funded by the Spanish Government under contract DPI2010-18449, and by a Juan de la Cierva contract supporting the second author.

\section{References}

[1] Bhattacharya, S., Hatwal, H., Ghosh, A.: Comparison of an exact and an approximate method of singularity avoidance in platform type parallel manipulators. Mechanism and Machine Theory 33(7), 965-974 (1998)

[2] Bohigas, O., Zlatanov, D., Ros, L., Manubens, M., Porta, J.: Numerical computation of manipulator singularities. In: Proc. of the IEEE Int. Conf. on Robotics and Automation (2012)

[3] Bosscher, P., Riechel, A., Ebert-Uphoff, I.: Wrench-feasible workspace generation for cable-driven robots. IEEE Trans. on Robotics 22(5), 890-902 (2006)

[4] Cortés, J., Siméon, T.: Probabilistic motion planning for parallel mechanisms. In: Proc. of the IEEE Int. Conf. on Rob. and Aut., vol. 3, pp. 4354-4359 (2003)

[5] Dasgupta, B., Mruthyunjaya, T.: Singularity-free path planning for the Stewart platform manipulator. Mechanism and Machine Theory 33(6), 711-725 (1998)

[6] Dash, A.K., Chen, I.M., Yeo, S.H., Yang, G.: Workspace generation and planning singularity-free path for parallel manipulators. Mechanism and Machine Theory 40(7), 776-805 (2005)

[7] Henderson, M.E.: Multiple parameter continuation: Computing implicitly defined $k$-manifolds. Int. J. of Bifurcation and Chaos 12(3), 451-476 (2002)

[8] Hubert, J.: Manipulateurs parallèles, singularités et analyse statique. Ph.D. thesis, École Nationale Supérieure des Mines de Paris (2010)

[9] Li, H., Gosselin, C., Richard, M., St-Onge, B.: Analytic form of the sixdimensional singularity locus of the general Gough-Stewart platform. ASME Journal of Mechanical Design 128, 279-288 (2006)

[10] Merlet, J.: Parallel robots. Springer-Verlag (2006)

[11] Merlet, J.P., Gosselin, C.: Springer Handbook of Robotics, chap. Parallel Mechanisms and Robots, pp. 269-285. Springer (2008)

[12] Porta, J.M., Jaillet, L.: Path planning on manifolds using randomized higherdimensional continuation. In: D. Hsu, V. Isler, J.C. Latombe, M. Lin (eds.) Algorithm. Found. of Robotics IX, STAR, vol. 68, pp. 337-353. Springer (2011)

[13] Russell, S.J., Norvig, P.: Artificial Intelligence: A Modern Approach. Prentice Hall (2003)

[14] Sen, S., Dasgupta, B., Mallik, A.K.: Variational approach for singularity-free path-planning of parallel manipulators. Mechanism and Machine Theory 38(11), 1165-1183 (2003)

[15] Voglewede, P., Ebert-Uphoff, I.: Overarching framework for measuring closeness to singularities of parallel manipulators. IEEE Trans. on Robotics 21(6), $1037-1045$ (2005) 